\title{
Rule-based fuzzy classifier for spinal deformities
}

\author{
Sibel Birtane ${ }^{\mathrm{a}, *}$ and Hayriye Korkmaz ${ }^{\mathrm{b}}$ \\ ${ }^{a}$ Department of Computer Programming, Vocational School, Istanbul Arel University, Istanbul, \\ Turkey \\ ${ }^{b}$ Department of Electrical and Electronics Engineering, Faculty of Technology, Marmara University, \\ Istanbul, Turkey
}

\begin{abstract}
In this paper, 2-steps software using image processing and enhancement technologies is developed to obtain a scoliosis patient's spine pattern from 2D coronal X-Ray images without manual land marking. Then, a Rule-based Fuzzy classifier is implemented on those images to classify the spine patterns using the King-Moe classification approach.
\end{abstract}

Keywords: Image processing, scoliosis, king-moe type classification, fuzzy logic, computer aided classification, rule-based classification

\section{Introduction}

Today, imaging technologies in medical fields reached a point where outputs are of much higher quality. This advancement allows for automated assessments of the images by using ruled-based decision support systems or computer-aided diagnostics/diagnostic supporting software instead of humans (physician or some other specialized healthcare professional) using traditional methods. Studies in image processing and medical informatics diminish the role of human factor in visual assessments while increasing the role of computer control [1-9].

Scoliosis is a common type of spinal deformity [2]. In these cases, the patient is periodically checked, and the degree of curvature is reassessed each time carefully. In each phase, radiological assessments are utilized; the surgical operation, on the other hand, is quite expensive. The physician's experience is a significant factor. It is possible to uncover the reason of deformity, its model, degree and the rate of vertebral shift through X-Rays. The classifications and various measurements in scoliosis cases are important to determine the treatment method used by the physician, the method of operation, which instruments to be used during the operation and whether the treatment will be beneficial or not [10-12].

\footnotetext{
*Corresponding author: Sibel Birtane, Department of Computer Programming, Vocational School, Istanbul Arel University, Istanbul, Turkey. Tel.: +90 54331939 01; Email: sibelbirtane@arel.edu.tr.
}

0959-2989/14/\$27.50 @ 2014 - IOS Press and the authors. 
Table 1

The methods and success of similar studies discussed in literature

\begin{tabular}{lllll}
\hline Study & Classification System & Method & Number of Models & Success Rate \\
\hline H. Lin [21] & King-Moe Classification & Neural Network & 17 & $66 \%$ \\
\hline H. Lin [22] & King-Moe Classification & $\begin{array}{l}\text { Neural Network } \\
\text { (Bezier) }\end{array}$ & 25 & $65 \%$ \\
\hline H. Lin and D. Sucato [13] & King-Moe Classification & Neural Network & 37 & $72 \%$ \\
\hline N. Mezghani ve friends. [16] & Lenke Classification & Rule-Based & 603 & $93 \%$ \\
\hline Proposed System & King-Moe Classification & $\begin{array}{l}\text { Fuzzy Logic } \\
\text { Rule-Based }\end{array}$ & 25 & $80 \%$ \\
\hline
\end{tabular}

In the literature review, it is possible to see digitized mammograms, brain tomography, dental XRay and various orthopedic images in a computer environment, enhancement of these images, 3D modeling and computer-aided classifications using various methods [1,13-22].

Studies focusing on classification of digitized medical images mostly utilize artificial neural networks, genetic algorithms, fuzzy logic, correlation, regression, Bezier curves and K-means algorithms. In the selection of classification method, the required qualifications and quantifications are proven to be significant for accurate diagnosis based on the medical image $[9,13,16,18,21-24]$. The methods and success of studies on computer-aided diagnosis of spinal deformities are given in Table 1. As seen in Table 1, among the computer-aided classification methods, Lenke type has a higher success rate. It is because the spine is assessed in 3D. It was also observed that most of the classification studies are conducted on scoliosis models. Based on these findings, it is safe to conclude that the success rate of the proposed system is quite high when compared to other systems. [13,16,21,22]

\section{Materials and methods}

Spine is a long, strong and flexible part of the skeleton composed of vertebrae that lies in the centerline of the body, and carries and supports the weight of it. The spine, composed of the perfect engagement of 33 vertebrae bones with joints and ties, is recessed in neck and lumbar region and protruding in the dorsal area in a side view, and straight from the front as seen some pictures in literatures [10]. Scoliosis, on the other hand is the curvature of the spine to the right or left, as seen in Figure 1, on the thoracic or lumbar regions. In a patient suffering from scoliosis, an examination called orthoroentgenography is conducted. Orthoroentgenography is a radiologic examination of head, body and hips in a single film taken while patient is standing still. The degree of deformation is observed on the film $[11,12]$.

Three different classification methods are used in the assessment of spinal deformities in terms of its shape. The most common method known as "King-Moe" classification was developed in 1983 by taking the thoracic deformities into account [2]. "Lenke" type classification, on the other hand was published in 2001 to compensate for the shortcomings of the King-Moe classification method $[3,16]$. Finally, more practical and comprehensible PUMC (Peking Union Medical College) classification method is defined in 2005 [4,5,25]. 


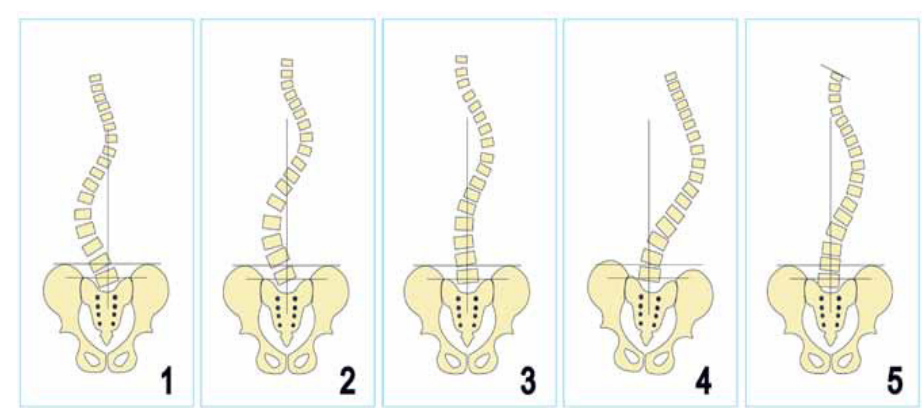

Fig. 1. King-Moe Classification models.

As mentioned before, Lenke type has a higher success rate. For Lenke type classification, both coronal and sagittal image of the spine is required. Since it is difficult to obtain these images of the patient, King-Moe classification model is chosen in this study where only the coronal image is sufficient [15-17]. Figure 1 shows the "King-Moe Classification" model composed of 5 types developed for modeling idiopathic scoliosis [2,26,27].

Type 1: There is a double curvature in an "S" shape. The lumbar (waist, lower) curvature is larger than the thoracic (chest, upper) curvature and/or lumbar curvature is less flexible than thoracic one.

Type 2: There is a double curvature in an " $\mathrm{S}$ " shape. Thoracic curvature is larger than or equal to lumbar curvature. Thoracic curvature is less flexible than the lumbar curvature. Lumbar curvature crosses the central sacral line.

Type 3: There is a single major thoracic curvature. The lower level of the curvature does not cross the midline.

Type 4: There is a single major long thoracic curvature. The vertebra L5 is centered over the sacrum. However, L4 tilts towards the convex side of the curvature.

Type 5: There is a dual structural curvature in the thoracic region. T1 tilts towards the convex side of the upper thoracic curvature. King types II and III are the most common groups where operations are performed frequently. [2,26]

\section{Software development}

The proposed software is composed of two steps as seen in Figure 2: 1) Image processing and enhancement and 2) Classification. Moreover, an interface as seen in Figure 3 is designed to allow interaction between all these processes and the user [28].

The study uses X-Ray images of scoliosis patients. In the test stage of fuzzy logic based classification system, 25 scoliosis models are used from the study by Lin H. and Sucato D. as well as a database composed of $10 \mathrm{X}$-Rays obtained from various public and private hospitals around Istanbul [16].

A system user interface seen in Figure 3 is designed as an interactive tool, and enables the following features: an X-Ray image can be opened from the existing files; user can monitor the image enhancement phases as well as their results. It also indicates the outcome of classification type obtained through Rule Based Fuzzy Classifier and all these results can be stored in a file. 


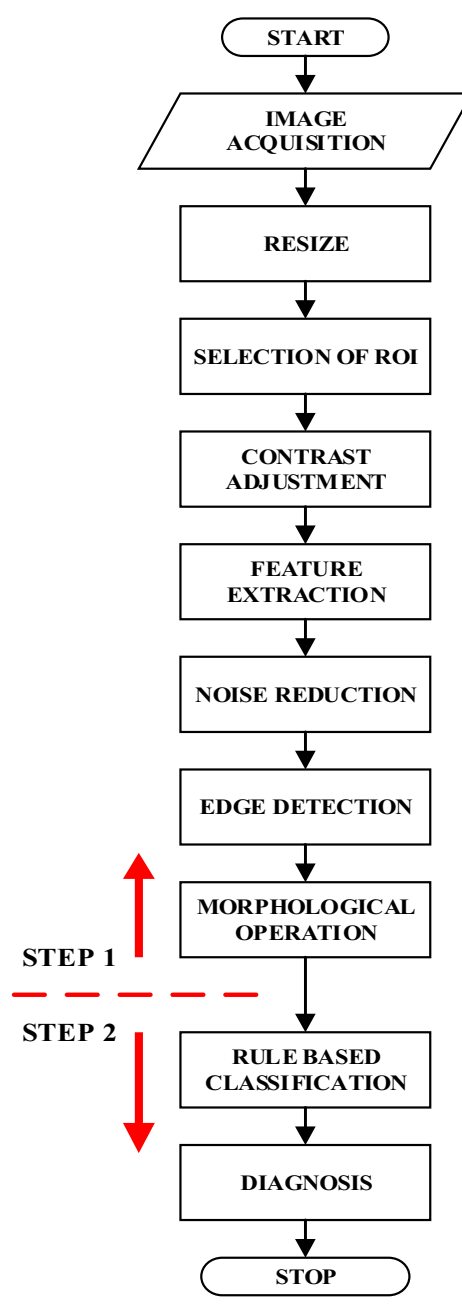

Fig. 2. Procedures of 2-steps software [28].

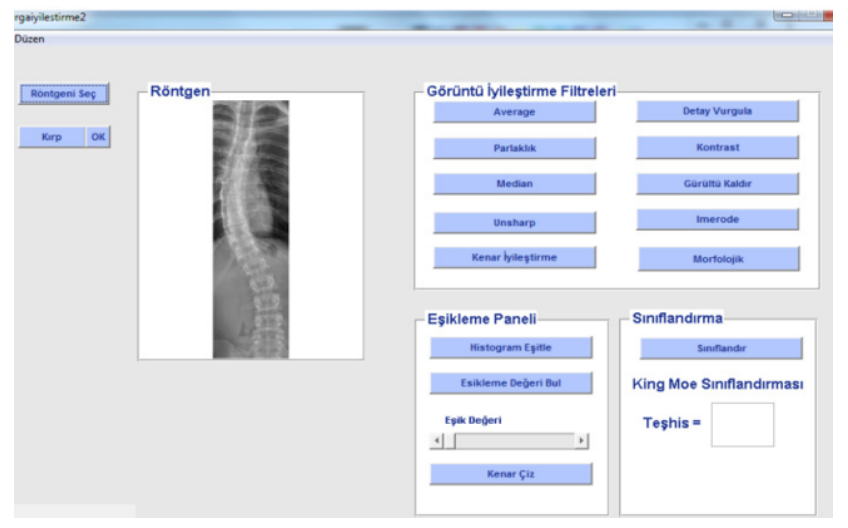

Fig. 3. Graphical user interface in Turkish [28]. 


\subsection{Image enhancement}

In the process from the X-Ray to obtaining the spinal pattern, a number of image enhancing techniques are used consecutively as seen in Figures 2 and 3. Image processing starts with the transfer of the image, and continues with the use of different techniques as required. There are many softwares in image processing field. However since image processing is based on mathematical calculations, Matlab Image Processing Toolbox is used during image processing and enhancement phases of this study in addition to Fuzzy Logic Toolbox in classification phase; Matlab GUI Builder is also used to make these processes interactive for the user.

To be able to use X-rays in classification process, only spine is required. To achieve this, a series of processes are applied as seen in Figure 2: Initially, original image is transferred to the user-interface and then cropped manually. After that, a scaling is performed to create identically-sized X-Rays obtained from different machines as shown in Figure 4(a). To separate unwanted images of ribs, contrasts settings are adjusted by adaptive histogram thresholding after selecting the appropriate area as shown in $4 \mathrm{~b}$. To be able to subtract the background image and detect the spine pattern only, a number of filters are performed consecutively shown in Figure 4(c). Morphological masking functions are utilized to fill the missing pixel by using "imfill holes" function shown in Figure 4(d). Canny filters are used to locate the edges of obtained spine images since it detects the changes in the images better than the Sobel filters. Next, morphological procedures are performed again to avoid the discontinuities of the spine pattern. Finally, the outline of the spine is obtained as shown in Figure 4(e).

In this study, the spine's outline is determined automatically instead of manual marking (land marking) and finding the most suitable curvature passing through these marks as done in studies [8] and [29]. The data about the curve seen in Figure 4(e) are used in the next stage, the classification.

Furthermore, Hong Lin's study in 2008, [22] benefits from 3D modeling by using Bezier curves to identify the curvatures in the images obtained from 37 patients' spinal X-Rays. While reviewing the literature, some classification methods applied in the field was observed. In one of the methods, manual markings are used to detect the spine curve. It is a much harder and time-consuming method. In the proposed system, a simple distance measurement method is used to determine how much the curvature deviates from the sacral line which is regarded as a reference for healthy spinal image. This method is a much simpler, automatic and comprehensible than the methods used in other studies.

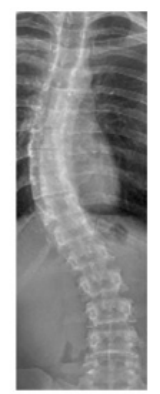

(a)

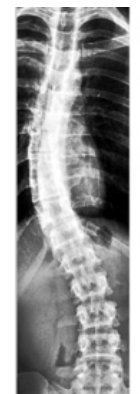

b)

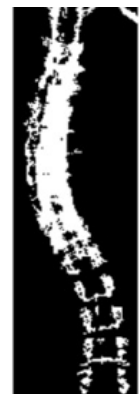

c)

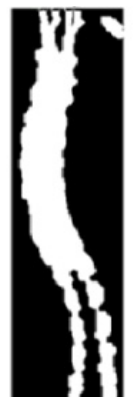

d)

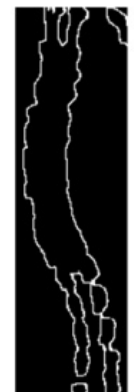

e)

Fig. 4. Reconstruction steps of a spine pattern. 


\subsection{Fuzzy logic classifier}

When the outline of the spine pattern is attained, it is placed on a Cartesian coordinate plane as seen in Figure 5 to obtain the inputs that will be used during classification. In this plane, initially two limit points (the outermost left and right endpoint) marked with P1 and P2 as seen in Figure 5 are identified automatically; then other parameters are established in relation to these points. Six input parameters are defined to use in rule-based classification system: Xmax, Ymax, Xmin, Ymin, region and length. These can be described as:

$\mathrm{Xmax}$; the highest $\mathrm{x}$ pixel value of the spinal curvature in region 1 through a shift from the reference line over the $\mathrm{x}$ plane;

Ymax; $y$ pixel value of the point defined for Xmax;

$\mathrm{Xmin}$; the lowest $\mathrm{x}$ pixel value of the spinal curvature in region 2 through a shift from the reference line over the $\mathrm{x}$ plane;

Ymin; y pixel value of the point defined for Xmin;

Region, the variable that indicates whether the largest region between the pattern of "S" shaped spine and the reference line lies on the right side of the reference line (positive, Region 1) or the left (negative, Region 2);

$\Delta X=|X m a x-X r e f|-|X m i n-X r e f|$, If $\Delta X>=0$ then region is positive else region is negative.

Length, the value of distance (along the $\mathrm{y}$ axis) on how much continuity the spinal pattern shows over an imaginary reference line drawn vertically to the sacral point.

In this study, since the parameter derived from the spinal pattern varies in each person, (the number of pixels covered by whole spine along the y axis in total; also how many pixels does the neck, chest or lumbar regions cover) and also varies depending on the deformation type, the portions of the spine cannot be determined certainly. Therefore, a commonly utilized fuzzy modeling technique-Mamdani type algorithm which is considered as intuitive and similar to human behavior is used.

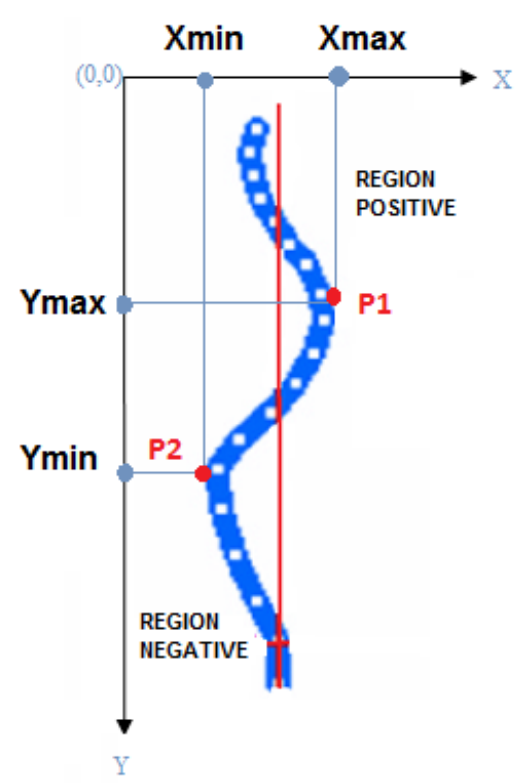

Fig. 5. Definition of Input variables used for classification on the Cartesian coordinate plane [28]. 


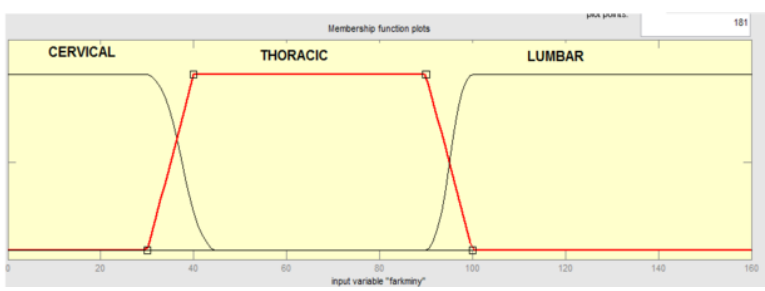

(a)

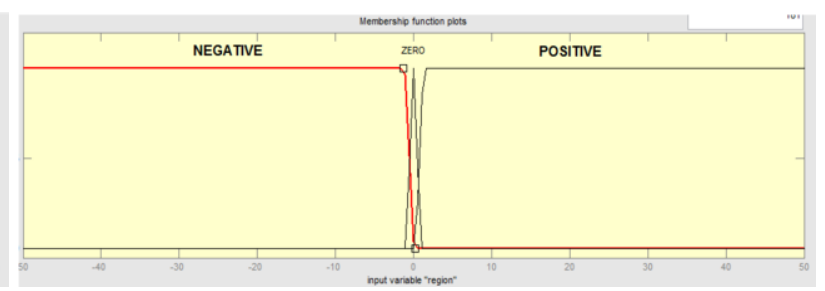

(b)

Fig. 6. Membership functions a) for variable "Length" b) for variable "Region".

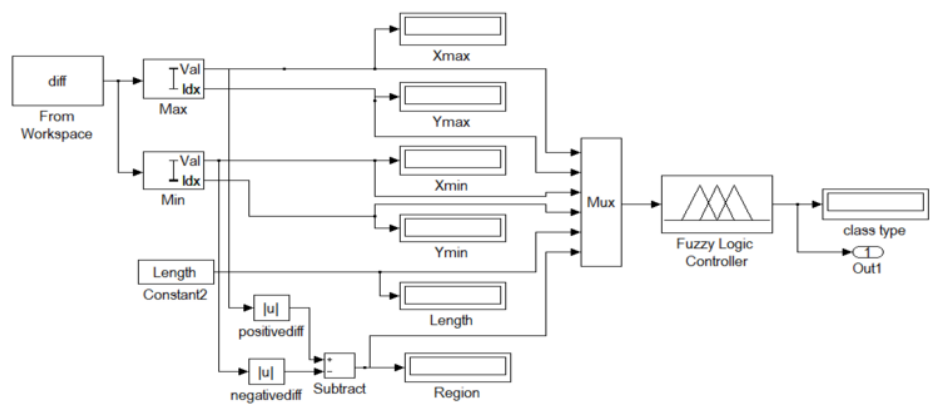

Fig. 7. Simulink block diagram.

In the first step, inputs are converted into fuzzy values by identifying membership functions as seen Figures 6(a) and 6(b). [28]. In the second step, the following rules are developed and applied based on King-Moe classification system by using the obtained fuzzy variables:

- If (Xmin is negative) and (Ymin is lumbar) and (region is region 2) then (class is type 1)

- If (Xmax is positive) and (Ymax is thoracic) and (region is region 1) then (class is type 2)

- If (Xmax is positive) and (Ymax is thoracic) and (Xmin is zero) and (Ymin is lumbar) and (length is thoracic) then (class is type 3 )

- If (Xmax is positive) and (Ymax is thoracic) and (Xmin is zero) and (Ymin is not thoracic) and (length is not thoracic) then (class is type 4)

- If (Xmax is positive) and (Ymax is not lumbar) and (Xmin is negative) and (Ymin is cervical) and (length is not cervical) then (class is type 5)

In the last step, with the inference system created in Simulink environment, the outputs are defuzzified and then converted into a single output by using weighted mean method seen in Figure 7.

\section{Results and discussion}

The Rule Based Fuzzy classifier achieves $80 \%$ success rate on scoliosis models as seen in Table 2 while $50 \%$ success rate is obtained on real scoliosis X-Rays shown in Table 3.

When reviewing other studies in the literature, it was observed that the most common form of classification is done on the scoliosis models. Accordingly, the success rate of the proposed system on the models is quite high when compared to other systems. However, it is impossible to compare the success rate of real X-rays with other studies which are using models. [13,18,21,22]

At the end of the classification process, the success rate on real X-Rays turned out to be lower than scoliosis models. A higher success rate of the proposed system depends on a continuous spinal pattern 
Table 2

Scoliosis models using RBFC success rates

\begin{tabular}{|c|c|c|c|c|}
\hline Type & $\begin{array}{l}\text { Number of } \\
\text { Models }\end{array}$ & $\begin{array}{l}\text { Correctly } \\
\text { Instances }\end{array}$ & $\begin{array}{l}\text { Incorrectly } \quad \text { Classified } \\
\text { Instances }\end{array}$ & $\begin{array}{l}\text { Incorrect Classification } \\
\text { Result }\end{array}$ \\
\hline TYPE 1 & 2 & 1 & 1 & TYPE 2 \\
\hline TYPE 2 & 2 & 1 & 1 & TYPE 1 \\
\hline TYPE 3 & 2 & 2 & 0 & ---- \\
\hline TYPE 4 & 2 & 0 & 2 & TYPE 1 , TYPE 3 \\
\hline TYPE 5 & 2 & 1 & 1 & TYPE 1 \\
\hline $\begin{array}{l}\text { Total Numbered of } \\
\text { Samples }\end{array}$ & 10 & 5 & 5 & ----- \\
\hline$(\%)$ & $100 \%$ & $50 \%$ & $50 \%$ & ----- \\
\hline
\end{tabular}

Table 3

X-Rays using RBFC success rates

\begin{tabular}{llllll}
\hline Type & $\begin{array}{l}\text { Number } \\
\text { Models }\end{array}$ & $\begin{array}{l}\text { of } \\
\text { Instances }\end{array}$ & $\begin{array}{l}\text { Correctly } \\
\text { Insified }\end{array}$ & $\begin{array}{l}\text { Incorrectly } \\
\text { Instances }\end{array}$ & $\begin{array}{c}\text { Classified } \\
\text { Incorrect Classification } \\
\text { Result }\end{array}$ \\
\hline TYPE 1 & 5 & 4 & 1 & TYPE 4 \\
\hline TYPE 2 & 5 & 5 & 0 & --- \\
\hline TYPE 3 & 5 & 4 & 1 & TYPE 2 \\
\hline TYPE 4 & 5 & 4 & 1 & TYPE 2 \\
\hline TYPE 5 & 5 & 3 & 2 & TYPE 2, TYPE 3 \\
\hline $\begin{array}{l}\text { Total Numbered } \\
\text { Samples }\end{array}$ & of & 25 & 20 & 5 & ---- \\
\hline \begin{tabular}{l}
$(\%)$ \\
\hline
\end{tabular} & $100 \%$ & $80 \%$ & $20 \%$ & ----- \\
\hline
\end{tabular}

of high-quality. This is because actual X-rays are not acquired from a single device and hospital, and the resolution and the distance between the patient and the device is different. The sizes of the X-ray images acquired are not the same, too.

Furthermore, the size of the spine differs in each patient and has no specific standard. As a result, some difficulty is experienced in image processing part of the study. When all these factors are taken into consideration, the reason of low rate of success in actual X-rays can be explained with the failure to improve the actual X-rays optimally rather than the classification phase. This limitation reduces the success rate of the classification process as well.

By applying the image enhancement steps developed and given in Figure 2 on images acquired from just a single device can be continued in the study. Thus, to obtain a higher success rate is very likely.

\section{References}

[1] İ. Kahraman, V. Bayram, E. Ercan and B. Kirllmaz, Implementation of medical image processing in the measurement of blood vessels width change, AkademikBilişim (2010), 557-560.

[2] H.A. King, J.H. Moe, D.S. Bradford and R.B. Winter, The selection of fusion levels in thoracic idiopathic scoliosis, J. Bone Joint Surg. Am. 65 (1983), 1302-1313.

[3] L.G. Lenke, Lenke classification system of adolescent idiopathic scoliosis: Treatment recommendations, AAOS Instructional Course Lectures 54 (2005), 537-542.

[4] G. Qiu, J. Zhang, Y. Wang, H. Xu, J. Zhang and X. Weng, A new operative classification of idiopathic scoliosis: A peking union medical college method, Spine (Phila Pa 1976) 30 (2005), 1419-1426. 
[5] G. Qiu, J. Zhang, Y. Wang, H. Xu, J. Zhang and X. Weng, Comparison of reliability between the PUMC and lenke classification systems for classifying adolescent idiopathic scoliosis, Spine(Phila Pa 2976) 33 (2008), E836-842.

[6] J. Novosad, F. Cheriet, Y. Petit and H. Labelle, Three-dimensional (3-D) reconstruction of the spine from a single x-ray image and prior vertebra models 51 (2004), 1628-1639.

[7] J. Boisvert, F. Cheriet, X. Pennec, N. Ayache and H. Labelle, A novel framework for the 3D analysis of spine deformation modes, Stud. Health Technol. Inform. 123 (2006), 176-181.

[8] S. Kadoury and H. Labelle, Classification of three-dimensional thoracic deformities in adolescent idiopathic scoliosis from a multivariate analysis, Eur. Spine J. 21 (2012), 40-49.

[9] S. Negrini, A. Negrini, S. Atanasio and G.C. Santambrogio, Three-dimensional easy morphological (3-DEMO) classification of scoliosis, Part I. Scoliosis 1 (2006), 20.

[10] K.M. Bayraktar, The surgical treatment of adolescent idiopathic scoliosis posterior instrumentation and fusion results in, Expertise Thesis, Haseki Training and Research Hospital, Orthopaedics and Traumatology Klinic, 2008.

[11] Ö. Ecerkale, Posture analysis in the evaluation of the results orthorontgenogram with symmetrigraf, M.Sc. Dissertation, Okmeydanı Training And Research Hospital, Physical Therapy And Rehabilitation Clinic, 2006.

[12] C. Y1lmaz, Surgical treatment of adolescent idiopathic scoliosis, M.Sc .Dissertation, Ankara University, 2001.

[13] H. Lin and D. Sucato, Clustering property of spinal deformity classification with simplified 3-D model and principal component analysis, Proceedings of the 5th International Conference on Information Technology and Application in Biomedicine, in Conjunction with the 2nd International Symposium \& Summer School on Biomedical and Health Engineering, 2008, 358-361.

[14] N. Mezghani, R. Chav, L. Humbert, S. Parent, W. Skalli and J. Guise, A computer-based classifier of three-dimensional spinal scoliosis severity, International Journal of Computer Assisted Radiology and Surgery 3 (2008), 55-60.

[15] N. Mezghani, P. Phan, A. Mitiche and H. Labelle, A computer-aided method for scoliosis fusion level selection by a topologicaly ordered self-organizing Kohonen network, 2010 International Conference on Pattern Recognition, 2010, 4012-4015.

[16] N. Mezghani, P. Phan, H. Labelle, C. Aubin and J. Guise, Computer-aided lenke classification of scoliotic spines, World Academy of Science, Engineering and Technology, International Science Index 293 (2012), 646 -649.

[17] N. Chockalingam, P. Dangerfield and G. Giakas, Computer-assisted Cobb measurement of scoliosis, Eur. Spine Journal 11 (2002), 353-357.

[18] U. Özbek, Investigation of Dental radiographs by using computer-based image processing techniques and classification using ANN, M.Sc. Dissertation, Selçuk University, 2006.

[19] H. Kim, H.J. Tan, S. Ishikawa and T. Shinomiya, Automatic detection of spinal deformity based on statistical features from the moire topographic images, Computing 8 (2009), 72-78.

[20] F. Berryman, P. Pynsent, J. Fairbank and S. Disney, A new system for measuring three-dimensional back shape in scoliosis, Eur. Spine J. 17 (2008), 663-672.

[21] H. Lin, Identification of spinal deformity classification with total curvature analysis and artificial neural network, Proceedings of the 2005 IEEE Engineering in Medicine and Biology 27th Annual Conference 6 (2005), 6168-6171.

[22] $\mathrm{H}$. Lin, Identification of spinal deformity classification with total curvature analysis and artificial neural network, IEEE Transactions on Biomedical Engineering 55 (2008), 376-382.

[23] M.S. Ozerdem and V. Akpolat, Classification of bone density with using neural networks signal processing and communications applications, IEEE 15th Signal Processing and Communications Applications, 2007, 1-5.

[24] M. Gstoettner, K. Sekyra, N. Walochnik, P. Winter, R. Wachter and C.M. Bach, Inter-and intraobserver reliability assessment of the cobb angle: Manual versus digital measurement tools, Eur. Spine J. 16 (2007), 1587-1592.

[25] W.B. Sheng, X.F. Zheng, H.L. Guo, Y.L. Zhan, E.D. Mai, G.L. Jin, L.T. Pu, J. Sheng and Q. Deng, Intraobserver and interobserver reliability of the king, lenke, and PUMC classification systems for idiopathic scoliosis, PubMed 89 (2009), $1047-1052$.

[26] J.P. Dormans, Pediatric Orthopaedics: Core Knowledge in Orthopaedics, 1st edi., Elsevier Mosby, Philadelphia, 2005 , pp. 265-278.

[27] E. Oğuz, Ş. Ekinci and Ö. Erşen, Review of radiological assessment and classification systems of adolescent idiopathic scoliosis, Journal of the TOTBID 12 (2013), 73-82.

[28] S. Birtane, Software development for classification of spinal deformities, M.Sc. Dissertation, Institute of Pure and Applied Sciences, Marmara University, 2013.

[29] J.R. Dimar, L.Y. Carreon, H. Labelle, M. Djurasovic, M. Weidenbaum, C. Brown and P. Roussously, Intra-and interobserver realibility of determining radiographic sagittal parameters of the spine and pelvis using a manual and a computer-assisted methods, Eur Spine J. 17 (2008), 1373-1379. 